\author{
Johannes M. Froehlich \\ Christian Waldherr \\ Christoforos Stoupis \\ S. Mehmet Erturk \\ Michael A. Patak
}

\section{MR motility imaging in Crohn's disease improves lesion detection compared with standard MR imaging}

Received: 19 November 2009

Revised: 12 January 2010

Accepted: 11 February 2010

Published online: 9 April 2010

(C) European Society of Radiology 2010

J. M. Froehlich · C. Waldherr .

C. Stoupis $(\bowtie) \cdot$ M. A. Patak

Institute of Diagnostic, Interventional

and Pediatric, Radiology, Inselspital,

University Hospital,

Freiburgstrasse,

3010 Bern, Switzerland

e-mail: c.stoupis@spitalmaennedorf.ch

Tel.: +41-31-6322111

Fax: +41-31-6324874

S. M. Erturk

Department of Radiology, Sisli Etfal

Hospital,

Istanbul, Turkey

\begin{abstract}
Objective: To evaluate retrospectively in patients with Crohn's disease (CD) if magnetic resonance (MR) motility alterations correlate with CD typical lesions leading to an increased detection rate. Methods: Forty patients with histologically proven $\mathrm{CD}$ underwent $\mathrm{MR}$ enterography (MRE), including coronal cine sequences (cine MRE), in addition to the standard CD MR protocol. Two blinded readings were performed with and without cine MRE. Locations presenting motility alterations on the cine sequences were analysed on standard MRE for CDrelated lesions. This was compared with a second reading using the standard clinical MRE protocol alone.
\end{abstract} Results: The number of lesions localised by cine MRE and identified on standard MRE compared with standard MRE alone were 35/24 for wall thickening ( $p=0.002), 24 / 20$ for stenoses $(p=0.05), 17 / 11$ for wall layering $(p=0.02), 5 / 3$ for mucosal ulcers $(p=0.02)$ and $21 / 17$ for the comb sign $(p=0.05)$. Overall, cine MRE detected 35 more CD-specific findings than standard MRE alone $(124 / 89 ; p=$ 0.007 ) and significantly more patients with CD-relevant MR findings (34/28; $p=0.03)$. Conclusion: CD lesions seem to be associated with motility changes and this leads to an increased lesion detection rate compared with standard-MRE imaging alone.

Keywords MRI - Small bowel · Crohns disease $\cdot$ Motility $\cdot$ Peristalsis

\section{Introduction}

Diagnosis of Crohn's disease (CD) is based on a combination of clinical, laboratory, histological and imaging findings. No single diagnostic method alone allows unequivocal diagnosis [1]. Imaging of the localisation and distribution of pathological features provides supportive evidence for the diagnosis of $\mathrm{CD}$. Over the last decade new therapeutic strategies have been developed that allow the clinician to tailor therapy to the individual form of CD. The effectiveness of these treatments depends on accurate diagnosis of the nature and extent of disease. Today, magnetic resonance enterography (MRE) is one of the mainstays in the evaluation of $C D$. A wide variety of studies have proved an increase in sensitivity and specificity of MRE by focusing on the evaluation of static images [2-4]. MRE, however, allows not only the static display of morphology but also it can be combined with ultrafast imaging techniques (cine MRE) for analysis of bowel motility [5]. Furthermore, it is known from the literature, specifically from that on fluoroscopy, that CD-related lesions can lead to bowel motility disorders $[2-4,6-9]$. There are notions in the literature discussing the value of dynamic MR fluoroscopy but no structured study on motility has been performed [10,11]. Previous studies limited their evaluation either to the terminal ileum [12] or retrospectively correlated motility changes with longitudinal ulcers in a total of six patients [13].

The aim of the study, therefore, was to evaluate whether patients with CD demonstrate MR-detectable motility alterations in the affected small bowel and whether these motility changes might lead to an increased lesion detection rate. 


\section{Materials and methods}

Patients

The institutional review board approved this retrospective study, waiving the need for patient consent. The inclusion criteria were biopsy-proven $\mathrm{CD}$ and that the patient was in an active disease state at the time of MR imaging (MRI). An active disease state was defined as the presence of classic clinical symptoms such as fever, abdominal pain and diarrhoea, in combination with an increased $\mathrm{CD}$ activity index (CDAI) score $\geq 150[11,12]$ determined from the patients' files by the study coordinator $(\mathrm{CW})$. Exclusion criteria were the lack of histologically proven $\mathrm{CD}$, clinically inactive disease or a CDAI $<150$. Forty patients (22 men, 18 women; mean age 38 years, range 18 86 years) were included in the study.

\section{Patient preparation and MRI}

The applied MRE protocol included pre-examination fasting for $8 \mathrm{~h}$ and an oral uptake of $1,000 \mathrm{ml}$ of $3 \%$ mannitol (30 g mannitol dissolved in 1,000 $\mathrm{ml}$ of tap water). Every patient was instructed to drink this aqueous solution continuously over a period of $1 \mathrm{~h}$ before the examination. The patients were in a supervised waiting room during the time of oral uptake of the contrast medium. Additionally, patients were asked to report any evidence of bloating, nausea or episodes of diarrhoea or vomiting or any other side effects of the oral contrast medium.

MRI was performed on a 1.5-Tesla whole-body MR system (Sonata; Siemens Medical Solution, Erlangen, Germany) using the body-array surface coil (Siemens Medical Solution, Erlangen, Germany) covering the whole abdomen/pelvis. The patient was placed in a prone position into the magnet to reduce the breathing-dependent displacement of the small bowel. After measurement of a standard localiser, several cine sequences were performed using a coronal two-dimensional (2D) true FISP (fast imaging with steady state precession) (a single-slice true FISP, field of view $400 \mathrm{~mm}$, matrix of $256 \times 256$, flip angle $50^{\circ}$, slice thickness $10 \mathrm{~mm}$, slice repetition time of $500 \mathrm{~ms}$, acquisition time of $17 \mathrm{~s}$, apnoea under maximal inspiration). The sequence had a temporal resolution of $500 \mathrm{~ms}$ indicating that every $0.5 \mathrm{~s}$ a new image on the same plane was acquired. This cine sequence was repeated stepwise over the entire abdomen from anterior to posterior in a coronal orientation without a gap in between.

Subsequent spasmolysis to reduce movement artefacts was accomplished by administering an intravenous (i.v.) bolus of $40 \mathrm{mg} \mathrm{N}$-butylhyoscine (Buscopan, Boehringer Ingelheim, Germany).

The static MRE protocol was then applied with the following pulse sequences (refer to Table 1 for parameters in detail): 3D True FISP, 2D T2-weighted (T2w) HASTE, repetition of three identical T1w 3D FLASH series, 20, 60, and $90 \mathrm{~s}$ after bolus intravenous administration of contrast medium $(0.1 \mathrm{ml}$ per $\mathrm{kg}$ body weight of dimeglumine gadobenate, MultiHance, Bracco Diagnostics, Milan, Italy), followed by a multi-slice T1w 2D FLASH $3 \mathrm{~min}$ after i.v. contrast medium. This static MRE corresponded to the standard protocol used in clinical practice [14].

\section{Image analysis}

The evaluation of the patients' images was done on a picture archiving and communication system (PACS; EasyVision, version 10.2, Philips Imaging Systems, Best, The Netherlands). A standardised evaluation sheet was used for all readings. The examinations were randomly arranged and blinded on the PACS by the study coordinator (C.W.) who did not participate in the readout. Two experienced readers (C.S., M.A.P.) evaluated the images alone and then in consensus. Both readers had more than 9 years' MR experience and both were blinded to all previous clinical or diagnostic data, but they were aware of the patient inclusion criteria.

Four separate readings took place, which were at least 1 month apart. Either the observers were given only the images of the static MRE sequences (static MRE) or the whole set of images including both the cine and the static MRE sequences (cine MRE). The reading was done firstly alone (once for static and once for cine MRE), then repeated in consensus (once static, once cine MRE).

The static MRE images were evaluated according to previously published criteria [14]. The readers assessed image quality and small bowel distension, as well as typical morphological and imaging patterns indicating CD. Image

Table 1 Acquisition parameters for the routine static MRE sequences

\begin{tabular}{llllllll}
\hline Sequence-name & Orientation & TR ms & TE ms & Flip angle ${ }^{\circ}$ & FOV in mm & Matrix & Slice thickness \\
\hline True FISP & Axial \& coronal & 3.8 & 1.9 & 57 & 400 & $512 \times 384$ & $6 \mathrm{~mm}$ \\
2D HASTE & Axial \& coronal & 1010 & 80 & 90 & 400 & $512 \times 512$ & $6 \mathrm{~mm}$ \\
i.v. administration of & $40 \mathrm{mg}$ N-butylhyoscine and contrast agent & (Gd-BOPTA, 0.1 & mmol/kg body weight) & \\
3D FLASH & Coronal & 2.5 & 1 & 20 & 400 & $288 \times 320$ & $1.3 \mathrm{~mm}$ \\
2D FLASH & Axial \& coronal & 111 & 4.8 & 70 & 400 & $512 \times 512$ & $6 \mathrm{~mm}$ \\
\hline
\end{tabular}



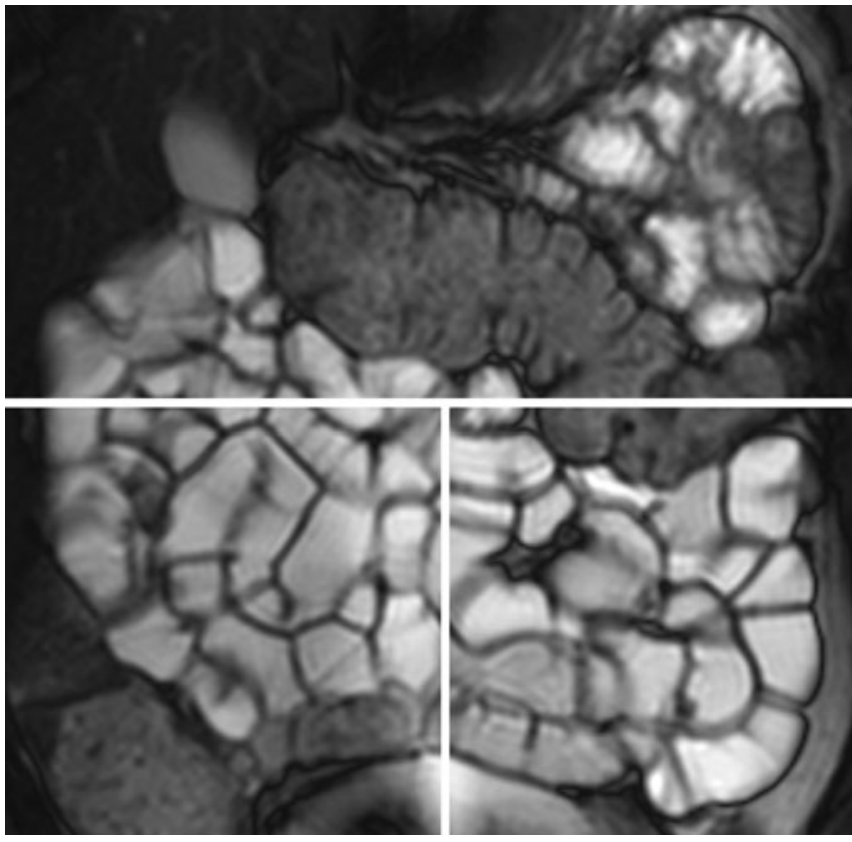

Fig. 1 Static MRE of a 36-year-old female patient with suspected small bowel CD. After oral preparation with $1,000 \mathrm{ml}$ of a $3 \%$ mannitol solution, the patient underwent a standardised MRI protocol. Image quality (graded on a three-point scale as good, moderate, or insufficient) and distension (complete or incomplete) were assessed at three different locations [upper abdomen (i.e. the jejunum), lower left abdomen for the ileum, and lower right abdomen for the terminal ileum] as indicated by the white lines. This patient (2D HASTE, TR 1,010, TE 80 , flip-angle $90^{\circ}$, matrix $512 \times$ 512 , slice-thickness $6 \mathrm{~mm}$ ) was scored as having good image quality and complete distension at all three locations

quality was graded on a three-point scale as good, moderate, or insufficient at three different locations (upper abdomen for the jejunum, lower left abdomen for the ileum, and lower right abdomen for the terminal ileum). Images were considered to be of good quality if the small bowel was clearly delineated and if they were free of hampering artefacts (Fig. 1). If some artefacts were present but did not reduce image interpretation, the image quality was deemed to be of moderate quality. In the case of unreadable images, the quality was classified as insufficient. The degree of small bowel distension was also analysed at three different locations (in the upper abdomen, mid-abdomen and terminal ileum). Distension was termed complete if the oral contrast medium permitted distinction between the wall and lumen along the entire length of the small bowel to the ileocaecal valve. If some part of the small bowel was collapsed, the distension was deemed incomplete. The following CDspecific morphological parameters were analysed on the static MRE images: small bowel wall thickening (bowel wall $\geq 4 \mathrm{~mm}$ ), stenoses (abnormal narrowing with or without bowel wall thickening, lumen $<2 \mathrm{~mm}$ ), prestenotic dilatation, layering of the bowel wall (thickened wall combined with alternating hyperintense and hypointense layers within the wall), ulcers (loss of integrity of the mucosa), the comb sign (multiple vessels on the mesenteric side of the small bowel), fistulas (abnormal connection or passageway between two bowel segments or between a small bowel structure and other hollow organs) and abscess (fluid collection with inflammatory changes in the surrounding mesenteric fat and pathological contrast enhancement in the wall).

Not every finding corresponds to a CD lesion by itself. Manifestations of CD generally rely on the joint presence of several of the above-mentioned features. Therefore CDspecific imaging findings of the same localisation are grouped to a single CD lesion.

The readout of the cine MRE series was done differently. Primarily, the images of the cine sequences were evaluated by displaying them as dynamic or movie sequences on the PACS with a frame rate of six images per second. This is about three times as fast as acquired. If a change in motility was identified on these dynamic cine sequences, it was cross-correlated with the identical localisation on the static MRE sequences to check and diagnose for pathological conditions. The cine sequences were thus used as indicators of pathological findings based on bowel motility alternations alone; pathological findings were finally interpreted on the static MRE images based on typical patterns for $\mathrm{CD}$ and imaging interpretation criteria as described above.

\section{Definition of motility alterations}

As stated above, motility was analysed using all cine sequences, thereby covering the entire small bowel. The analysis of the cine sequences was limited to qualitative criteria without measuring the peristaltic frequency. Normal motility was defined as a rhythmic motion of the small bowel walls with regularly alternating contractions and

Table 2 Overall image quality and qualitative rating of distension of standard clinical MRE in 40 patients with active CD

\begin{tabular}{llll}
\hline & Upper abdomen & Lower abdomen & Terminal ileum \\
\hline Good image quality & $70 \%$ & $78 \%$ & $78 \%$ \\
Moderate image quality & $23 \%$ & $20 \%$ & $13 \%$ \\
Insufficient image quality & $7 \%$ & $2 \%$ & $9 \%$ \\
Complete distension & $80 \%$ & $90 \%$ & $85 \%$ \\
Incomplete distension & $20 \%$ & $10 \%$ & $15 \%$ \\
\hline
\end{tabular}


dilatations of the lumen. This motility pattern is already known from previously published studies in healthy volunteers $[5,15,16]$. The motility changes were defined as zones of abnormal peristaltic motion and compared with surrounding bowel segments. Abnormal motility patterns were localised and classified according to the following definitions: paralysis (no sign of wall movement in the cine sequence), hypomotility (diminished or slow wall movement compared with surrounding bowel sections), or hypermotility (increased bowel wall movement compared with surrounding bowel segments). If paralysis was seen the presence of intraluminal pendular movement, i.e. the to-and-fro movement of the bowel content without any propelling or peristaltic action, was further evaluated. Finally, the qualitative motility status of the small bowel was categorised into two groups with the intent to correlate motility patterns with CD typical findings: Group A consisted of localisations with normal or hypermotility; Group B consisted of localisations with hypomotility or paralysis. Finally the presence and localisation of motility alterations were intraindividually correlated with typical findings for $\mathrm{CD}$ on the static MRE.

\section{Statistical analysis}

Patient data were characterised applying standard descriptive statistics. To compare the number of pathological findings from the static MRE with cine MRE, the number of CD lesions and number of patients with or without CD manifestations, the paired, two-sided Student's $t$-test was performed. A $p$ value of $<0.05$ was considered to be statistically significant. Inter-observer agreement was evaluated using linear-weighted Cohen's kappa statistics where a kappa value $>0.75$ was considered as excellent agreement, $0.4-0.75$ as fair to good agreement, and $<0.4$ as poor agreement [17]. The differences between the two motility groups with regard to the frequencies of MR findings mentioned above were investigated using Fisher's exact test.

\section{Results}

No adverse events or compliance problems were reported in the 40 examinations. The preparation scheme did not pose any problems; all patients were able to drink the entire amount of fluid and no gastrointestinal adverse effects such as vomiting or diarrhoea were mentioned by any of the patients. Bloating $(n=8)$ and nausea $(n=3)$ were mentioned in single cases, but did not lead to interruption of the preparation or MR examination.

\section{Image quality}

Static MRE image quality was scored as good by both readers in $70 \%$ in the upper abdomen, in $78 \%$ in the lower abdomen and in $78 \%$ in the terminal ileum (Fig. 1). The readers considered image quality to be moderate in $23 \%$ in the upper abdomen, in $20 \%$ in the lower abdomen and in $13 \%$ in the terminal ileum, and to be insufficient in $7 \%$ in the upper abdomen, in $2 \%$ in the lower abdomen and in $9 \%$ in the terminal ileum.

Small bowel distension was scored as complete in $80 \%$ of patients in the upper abdomen, in $90 \%$ in the lower abdomen, and in $85 \%$ in the terminal ileum (Fig. 1). It was therefore incomplete in $20 \%$ of the upper abdomen, $10 \%$ of the lower abdomen and $15 \%$ in the terminal ileum.

Of all examinations, $80 \%$ were without artefacts, $17 \%$ presented with motion artefacts and $20 \%$ with specific MR artefacts such as susceptibility, chemical shift or wraparound artefacts. Diagnostic image quality was hampered only seldomly despite this rather high incidence of artefacts.

An overview of these qualitative results is presented on Table 2.
Table 3 Number of CD-specific findings depicted by static MRE alone versus combined static MRE plus cine MRE in 40 patients with active $\mathrm{CD}$. The $p$ value indicates the statistical difference between the number of findings using the two-sided paired Student's $t$-test. The last column shows the kappa inter-observer agreement results for the two blinded readers

\begin{tabular}{|c|c|c|c|c|c|c|c|c|}
\hline & \multicolumn{2}{|l|}{ Reader 1} & \multicolumn{2}{|l|}{ Reader 2} & \multicolumn{4}{|l|}{ Consensus } \\
\hline & Cine MRE & Static MRE & Cine MRE & Static MRE & Cine MRE & Static MRE & $p$ value & $\begin{array}{l}\text { Inter-observer } \\
\text { agreement static vs cine }\end{array}$ \\
\hline Wall thickness & 31 & 22 & 35 & 21 & 35 & 24 & 0.002 & 0.9 \\
\hline Stenosis & 24 & 20 & 25 & 17 & 24 & 20 & 0.05 & 0.9 \\
\hline Layering & 17 & 11 & 9 & 10 & 17 & 11 & 0.02 & 0.8 \\
\hline Ulcer & 5 & 3 & 3 & 0 & 5 & 3 & 0.02 & - \\
\hline Comb sign & 21 & 17 & 21 & 15 & 21 & 17 & 0.05 & 0.8 \\
\hline Abscess & 2 & 0 & 1 & 0 & 2 & 0 & - & - \\
\hline Total findings & & & & & 104 & 75 & 0.02 & \\
\hline
\end{tabular}


Detection of motility alterations on the dynamic series

A total of 78 motility changes were detected in 29 patients, partitioned into the following patterns: 19 with paralysis (six upper abdomen, one lower abdomen, 12 terminal ileum), 49 with hypomotility (14 upper abdomen, 14 lower abdomen, 21 terminal ileum) and ten with hypermotility (six upper abdomen, one lower abdomen, three terminal ileum). All patients with paralysis showed a typical pendular fluid movement proximal to the paralytic zone. Using Fisher's exact test, the correlation of reduced motility (Group B) to CD-related findings was statistically significant for wall-thickening $(p<0.001)$, layering $(p=$ $0.002)$, stenosis $(p=0.002)$ and the comb sign $(p=0.009)$. Hypermotility (Group A) did not significantly correlate with any of the typical findings for CD.

\section{Typical findings for CD}

The total number of pathological findings detected in consensus (for single-reader results refer to Table 3 ) by cine MRE compared with static MRE corresponded to 35 versus 24 for small bowel wall thickening ( $p=0.002)$ (Fig. 2), 24 versus 20 for stenoses $(p=0.05), 17$ versus 11 for small bowel wall layering ( $p=0.02$ ), 5 versus 3 for mucosal ulcers $(p=0.02), 21$ versus 17 for the comb sign $(p=0.05)$ (Fig. 2a), and 2 versus 0 for abscesses. Both abscesses had a crossdiameter of less than $1 \mathrm{~cm}$ and were within a collection of inflamed loops leading to the impression of a further bowel loop, explaining in part why they were not found on static MRE, as re-analysis after exploitation of data confirmed (Fig. 3). Overall, cine MRE detected 29 more CD-specific findings than did static MRE (104 vs $75 ; p=0.02$ ).

Thirty-five CD lesions with the conjoint presence of various features as mentioned above were found on the cine MRE compared with 26 on the static MRE $(p=0.001)$.

Of these $35 \mathrm{CD}$-specific pathological areas found on cine MRE, only four of them showed no motility changes. There were also two locations where despite a change in motility no pathological features could be found.

Adding cine MRE to the reading 34 patients were finally identified as having typical lesions for CD whereas 25 patients were diagnosed based on the static reading $(p=$ $0.0007)$ alone.

The inter-observer agreement for both readers with a kappa value ranging from 0.8 to 1.0 depending on the rated feature was excellent (Table 3).

\section{Discussion}

The present study stipulates that motility is changed in affected small bowel locations in CD. Pathological alterations of the bowel wall apparently lead to hypomotility or even paralysis. Significantly more lesions could
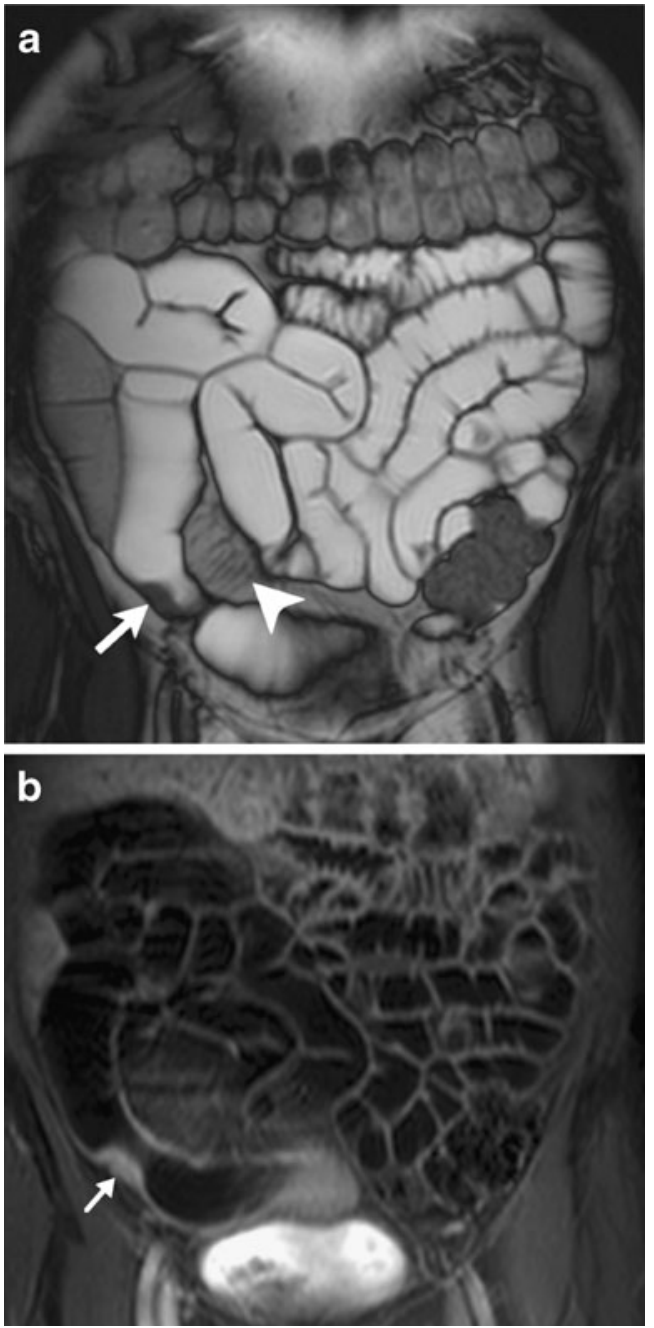

Fig. 2 MRI of a 28-year-old female patient with active CD. After oral preparation with $1,000 \mathrm{ml}$ of a $3 \%$ mannitol solution, the patient underwent a standardised MRI protocol. a Coronal True FISP (TR/ TE $3.8 / 1.9 \mathrm{~ms}$, flip angle $57^{\circ}$, matrix $512 \times 384$, slice thickness of $6 \mathrm{~mm}$ ) image shows segmental wall thickening (arrow), marked narrowing of the lumen of the terminal ileum, prestenotic dilatation, and the comb sign (arrowhead). b Corresponding post-contrast coronal 2D-FLASH (TR/TE $2.5 / 1 \mathrm{~ms}$, flip angle $20^{\circ}$, matrix $288 \times$ 320 , thickness $1.3 \mathrm{~mm}$ ) of the same location demonstrates the thickening and contrast enhancement of the affected small bowel wall (arrow). In this case, the cine MRE presented hypomotility of the affected segment (refer to online video clip)

thus be detected with the additional use of cine MR. Significantly more CD-specific pathological locations in the small bowel could be found translating in more patients with CD findings compared with evaluation of static images alone.

In general, our qualitatively rated distension of the small bowel with mean values of $85 \%$ attended similar ratings described in other MRE studies independantly of the distension agent used [3, 18, 19].

The use of MRI for purposes other than the acquisition of morphological images is surely not a new concept, but is 

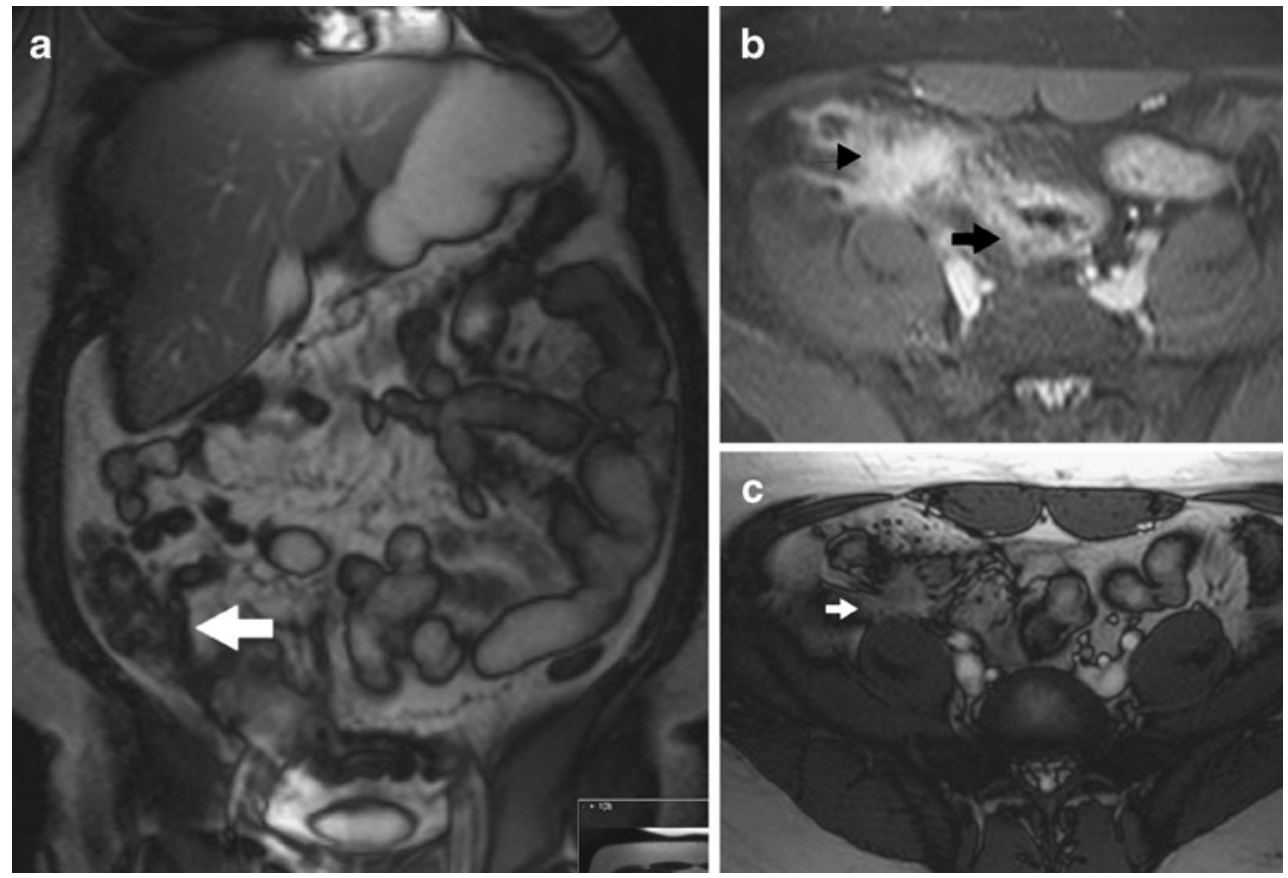

Fig. 3 MR imaging of a 20-year-old male patient with active CD. a Coronal 2D True FISP image shows unclear pathological area near the terminal ileum (arrow) with intermediate signal lacking clear borders (arrow) and lumen. This was interpreted as inflammation without abscess. b Corresponding axial 2D-FLASH image (TR/TE $2.5 / 1 \mathrm{~ms}$, flip angle $20^{\circ}$, matrix $288 \times 320$, thickness $6 \mathrm{~mm}$ ) after i.v. administration of contrast medium demonstrates the localised inflammation in the bowel wall (arrowhead) with diffuse enhance-

still rarely used in clinical routine. Cine imaging has long been used primarily for cardiac imaging [20]. Cine MRE of the small bowel has already been used to track motility changes in patients after abdominal surgery [16], to help establish an activity scoring system [12] or to rule out adhesion in abdominal imaging [13]. The present study, to our knowledge, is the first to add these sequences to an MRE static imaging protocol as a direct tool to increase the detection rate for CD-related lesions. Dynamic imaging of the small bowel is easy to apply on the different machine types, as similar cine sequences are already implemented in the software for cardiac imaging. Total examination time is only slightly prolonged for each patient.

It has to be emphasised, though, that cine MRE alone cannot be used as a diagnostic tool. It is only valuable for indicating motility alterations, while the specific CDrelated small bowel changes, such as wall thickness, stenoses, layering or contrast enhancement, can only be detected on the static images (Fig. 3). Comparison of cine MRE with static MRE cannot be performed, as cine MRE alone does not provide enough spatial resolution and contrast to make a final diagnosis.

Although there were single findings presenting with motility changes without static small bowel wall changes or vice versa, in most cases motility disorders were concordant with the pathological areas of the CD-related ment of the tissue around the terminal ileum (arrow). c Axial True FISP single-slice cine image (TR/TE $3.8 / 1.9 \mathrm{~ms}$, flip angle $57^{\circ}$, matrix $512 \times 384$, slice thickness of $6 \mathrm{~mm}$ ) across the same pathological finding shows the circular lesion close to the terminal ileum. The cine MRE images acquired in this patient demonstrate decreased motility in this section of the small bowel and a clear paraluminal fluid-filled lesion which was interpreted by both readers as an abscess (refer to online video clips)

findings. These false-positive/false-negative findings are difficult to interpret as there are not much data on normal small bowel motility in the sense of regular wall motion. We also know from manometry studies that the variability of motility can vary widely [21]. Possible explanations for lacking motility changes in CD-specific lesions might be (1) that these are not pronounced enough to be classified qualitatively, (2) that apparently not all CD-specific lesions show motility alterations, and (3) that motility alterations might also be missed in our setting. Reasons for hypomotility without CD-specific lesions might be a segment of bowel wall affected by a previous CD lesion inactive at the time of MR or a very early detection without any detectable morphological changes (in this case motility alterations would be a first sign of disease).

In our view, the increased $C D$ lesion detection rate of the combined reading method compared with the static MRE was surprising and might be explained as follows: apparently discrete affections of the small bowel already lead to motility changes, allowing earlier and improved imaging detection [22]. It seems that various CD specific alterations were interpreted differently or not as CDrelevant when lacking the additional information of dynamic imaging (Fig. 2). Moreover, technical reasons such as an increased number of images or differing signalto-noise or contrast-to-noise ratios might provide a further 
explanation. Finally, the total number of lesions typical of $\mathrm{CD}$ at 104 per 34 patients was rather high in accordance with their active state of disease.

The missing standard of reference was the major limitation of this study. Histopathological correlation of our MRI findings was not possible to accomplish within this study. Nevertheless, in general, sensitivity and specificity values of standard MRE present a high correlation with pathological findings using endoscopy $[23,24]$ as a standard of reference. In these MRE studies, high sensitivity/specificity levels of $92 \% / 67 \%$ and $83 \% / 100 \%$ for pathological changes are reported. Further prospective clinical studies are required to establish the correlation between peristaltic motion changes of the small bowel and their respective histopathological or surgical findings. Not all morphological features described in active $\mathrm{CD}$, such as mesenteric changes or enlarged lymph nodes, were rated within our study as they are already well described in the literature and most of these extraluminal changes presumably do not have an effect on small bowel motility $[4,11]$.

In conclusion, the use of cine MRE in patients suffering from $\mathrm{CD}$ proves the association of motility changes with small bowel wall changes. The inclusion of small bowel motility evaluation significantly increases the lesion detection rate for CD-related pathological findings compared with that of static MRE alone and thus could be of great importance for the management of patients with $\mathrm{CD}$.

\section{References}

1. Travis SP, Stange EF, Lemann M et al (2006) European evidence based consensus on the diagnosis and management of Crohn's disease: current management. Gut 55(Suppl 1):i16-i35

2. Masselli G, Casciani E, Polettini E, Gualdi G (2008) Comparison of MR enteroclysis with MR enterography and conventional enteroclysis in patients with Crohn's disease. Eur Radiol 18:438-447

3. Negaard A, Paulsen V, Sandvik L et al (2007) A prospective randomized comparison between two MRI studies of the small bowel in Crohn's disease, the oral contrast method and MR enteroclysis. Eur Radiol 17:2294-2301

4. Gourtsoyiannis NC, Grammatikakis J, Papamastorakis $\mathrm{G}$ et al (2006) Imaging of small intestinal Crohn's disease: comparison between MR enteroclysis and conventional enteroclysis. Eur Radiol 16:1915-1925

5. Froehlich JM, Patak MA, von Weymarn C, Juli CF, Zollikofer CL, Wentz KU (2005) Small bowel motility assessment with magnetic resonance imaging. J Magn Reson Imaging 21:370-375

6. Fidler J (2007) MR imaging of the small bowel. Radiol Clin North Am 45:317-331

7. Antes G, Lissner J (1983) Doublecontrast small-bowel examination with barium and methylcellulose. Radiology 148:37-40

8. Maglinte DD, Lappas JC, Kelvin FM, Rex D, Chernish SM (1987) Small bowel radiography: how, when, and why? Radiology 163:297-305
9. Sellink JL, Rosenbusch G (1981) ["The ten commandments" for enteroclysis or ten golden rules for proper enteroclysis technique]. Radiologe 21:366-376

10. Umschaden HW, Szolar D, Gasser J, Umschaden M, Haselbach H (2000) Small-bowel disease: comparison of MR enteroclysis images with conventional enteroclysis and surgical findings. Radiology 215:717-725

11. Prassopoulos P, Papanikolaou N, Grammatikakis J, Rousomoustakaki M, Maris T, Gourtsoyiannis N (2001) MR enteroclysis imaging of Crohn disease. Radiographics 21(Spec No):S161S172

12. Girometti R, Zuiani C, Toso F et al (2008) MRI scoring system including dynamic motility evaluation in assessing the activity of Crohn's disease of the terminal ileum. Acad Radiol 15:153-164

13. Kitazume Y, Satoh S, Hosoi H, Noguchi O, Shibuya H (2007) Cine magnetic resonance imaging evaluation of peristalsis of small bowel with longitudinal ulcer in Crohn disease: preliminary results. J Comput Assist Tomogr 31:876-883

14. Patak MA, von Weymarn C, Froehlich JM (2007) Small bowel MR imaging: 1.5 T versus $3 \mathrm{~T}$. Magn Reson Imaging Clin N Am 15:383-393 vii

15. Froehlich JM, Daenzer M, von Weymarn C, Erturk SM, Zollikofer CL, Patak MA (2009) Aperistaltic effect of hyoscine N-butylbromide versus glucagon on the small bowel assessed by magnetic resonance imaging. Eur Radiol 19:1387-1393

16. Patak MA, Froehlich JM, von Weymarn C, Breitenstein S, Zollikofer CL, Wentz KU (2007) Noninvasive measurement of small-bowel motility by MRI after abdominal surgery. Gut 56:1023-1025
17. Fleiss JL (1981) Statistical methods for rates and proportions. Wiley, New York

18. Ajaj W, Goyen M, Schneemann $\mathrm{H}$ et al (2005) Oral contrast agents for small bowel distension in MRI: influence of the osmolarity for small bowel distension. Eur Radiology 15:1400-1406

19. Ajaj W, Goehde SC, Schneemann H, Ruehm SG, Debatin JF, Lauenstein TC (2004) Dose optimization of mannitol solution for small bowel distension in MRI. J Magn Reson Imaging 20:648653

20. Pettigrew RI, Oshinski JN, Chatzimavroudis G, Dixon WT (1999) MRI techniques for cardiovascular imaging. J Magn Reson Imaging 10:590601

21. Camilleri M, Bharucha AE, di Lorenzo $\mathrm{C}$ et al (2008) American Neurogastroenterology and Motility Society consensus statement on intraluminal measurement of gastrointestinal and colonic motility in clinical practice. Neurogastroenterol Motil 20:12691282

22. Vermillion DL, Huizinga JD, Riddell RH, Collins SM (1993) Altered small intestinal smooth muscle function in Crohn's disease. Gastroenterology 104:1692-1699

23. Siddiki HA, Fidler JL, Fletcher JG et al (2009) Prospective comparison of stateof-the-art MR enterography and CT enterography in small-bowel Crohn's disease. AJR Am J Roentgenol 193:113-121

24. Lee SS, Kim AY, Yang SK et al (2009) Crohn disease of the small bowel: comparison of CT enterography, MR enterography, and small-bowel followthrough as diagnostic techniques. Radiology 251:751-761 